\title{
Nonlinear Optimal Control Analysis of Helicopter Maneuver Problems Using the Indirect Method
}

\author{
By Chang-Joo KIM, Jaewoo LeE, Young Hwan BYun and Yung Hoon YU \\ Department of Aerospace Information Systems Engineering, Konkuk University, Seoul, Korea
}

(Received May 7th, 2007)

\begin{abstract}
This paper deals with a nonlinear optimal control approach to helicopter inverse simulation. The reference trajectory is prescribed in prior, and the integral deviation from this trajectory is treated as an additional penalty cost to convert the system optimality to an unconstrained optimal control problem. The resultant two-point boundary value problem has been solved by a multiple-shooting algorithm. The nonlinear helicopter model in this study includes main rotor flap dynamics and a dynamic inflow model. The applications cover the inverse simulation for bob up, turn, and slalom maneuvers. This paper focuses on resolving the convergence issue using the indirect method, the main root causes of which are related to the inherent system instability of the helicopter and with poor initial guesses on state and costate variables. For this reason we will investigate the effect of the shooting node number on convergence and use a hybrid-model approach, where the optimal state and costate variables, calculated using the linear model, are used as initial guesses for those using the nonlinear model. The analyses show good convergence history and capability of tracking the prescribed trajectory. So the results in this paper can provide a valuable motivation for applying indirect methods to nonlinear helicopter flight mechanic analysis.
\end{abstract}

Key Words: Helicopter, Inverse Simulation, Nonlinear Optimal Control, Hybrid Model

\section{Nomenclature}

$f$ : system forcing function

$F$ : jumps at shooting nodes

$f_{\mathrm{CO}}$ : total operating cost function $\left(=f_{\mathrm{OP}}+f_{\mathrm{SC}}\right)$

$f_{\mathrm{OP}}$ : operating cost function

$f_{\mathrm{SC}}$ : penalty cost function for state constraints

$J$ : cost function

$M$ : Jacobian matrix of $F$

$p, q, r:$ angular velocity of rigid body

$Q, R$ : state and control weighting matrix

$s$ : function for state constraints

$u$ : control vector or longitudinal linear velocity

$U$ : feasible set of controls

$v, w$ : linear velocity in $y$-, $z$-direction

$x$ : state vector or $x$-position

$x_{\mathrm{E}}, y_{\mathrm{N}}, h: x-, y$-positions and altitude

$y, z:$ state vectors, used in control formulation

$Z$ : state and costate variables at shooting nodes

$\alpha$ : relaxation factor for Newton method

$\beta$ : blade flap angle

$\delta$ : main rotor and tail rotor controls

$\phi, \theta$ : roll angle and pitch angle

$\varphi$ : terminal cost function

$\lambda$ : costate variables or inflow ratio

$\mu$ : Lagrange multipliers for terminal conditions

$\psi$ : yaw angle or function for terminal conditions

Subscripts

$0,1 \mathrm{C}, 1 \mathrm{~S}$ : collective, 1 st cos and 1 st sin components

$\mathrm{R}$ : rigid body state

\author{
I: inflow state \\ F: main rotor flap state \\ TR: tail rotor \\ trim: trim condition
}

\section{Introduction}

Identification of flight characteristics is one of the most challenging problems in the domain of flight dynamics. Knowledge of these is usually gained after expensive and hazardous flight trials involving maneuvers. Therefore, reliable numerical methods have an essential role in providing quick and low-cost design iterations at early design stages and to give benchmark results in order to increase the productivity of flight tests. Whereas numerous studies on analyzing flight maneuvers can be found for conventional aircraft and space vehicles, publications on rotorcraft are very limited. This is related to the complexity of the analysis of the rotorcraft due to their inherent instability, coupling of rigid body dynamics and rotor dynamics, and the presence of many flight parameters and constraints.

Two common methods for analysis of helicopter maneuvers are the inverse simulation method and dynamic optimization. Inverse simulation finds controls that enable the helicopter to follow a prescribed flight path whose history is given as a function of time. Various inverse simulation methods have been developed over the last decade. ${ }^{1-5)}$ Among them, the integration inverse method, proposed by Hess et al., ${ }^{1)}$ has popularly been used because of its lower dependency on model structure. But numerical instability or oscillatory behavior in its solutions causes some difficulties in its 
application with the high-fidelity helicopter model. The time scale separation method by Matteis et al. ${ }^{2)}$ and the optimization method by $\mathrm{Celi}^{3)}$ are examples of techniques that eliminate numerical instabilities by relaxing the condition of exact trajectory following. However, further studies seem to be required to improve the robustness of this approach.

On the other hand, dynamic optimization ${ }^{6-8)}$ computes the controls, the state, and possibly the final time that minimize a cost function, subject to state equations and to various other constraints, as required by the problem at hand. There are basically two alternative strategies for the numerical solution of a dynamic optimization problem. ${ }^{4}$ The first is the indirect method where we first apply calculus of variation to find the necessary conditions which minimize a cost function. Then the problem is reduced to a TPBVP (twopoint boundary value problem) in the infinite dimension and a suitable MSM (multiple-shooting method) can be used to resolve it in the finite dimension. ${ }^{8-10)}$ The second is the direct method, ${ }^{6,7)}$ which uses the discretized or parameterized equation for state equations, constraint equations and a cost function in the finite dimension. The result becomes a nonlinear programming problem which can be solved by one of the efficient NLP (non-linear programming) methods. ${ }^{7)}$

An algorithm for dynamic optimization should be carefully selected by investigating various factors affecting convergence speed, convergence radius, flexibility, complexity, required computer storage, and accuracy, etc. ${ }^{4,7)}$ The direct method is the usual choice in application to helicopter flight dynamics because of its ease in handling state inequality constraints and its large convergence radius, which means a converged optimal solution can be obtained from a large range of different initial guesses. But the direct method has major drawbacks in its application to the helicopter. Whereas the indirect method can use a standard ODE solver, the direct method requires a prior parameterization during which important system characteristics can be lost. Another important issue with the direct method is the problem of large system size. When discretization is refined for high precision, analysis might require long computational time with a slow convergence rate. This is a major reason why most applications of the direct method to helicopter flight use a simplified model for rotor dynamics.

This paper applies the indirect method to analysis of helicopter maneuvers. For this reason, we focus on resolution of the following major issues with the indirect method. The first issue is the problem of how to handle trajectory constraints. If we use boundary constraints based on the prescribed trajectory, the solution needs prior knowledge of optimal trajectory to define the sub-arcs for active state constraints. This complicates the application of MSM even if we have enough information for optimal trajectory. The second issue with the indirect method is related to convergence speed and convergence radius. This method generally shows quick convergence and extremely high precision in the neighborhood of the optimal trajectory. However, it has a much smaller convergence radius than the direct method because of its high sensitivity to poor initial guesses on state and costate variables. The task of finding good initial guesses is often impossible because costate variables are not clear in their physical meanings. The next issue is the effect of system instability, which is important for an inherently unstable system like the helicopter. Time integration over a relatively large shooting segment generally leads to catastrophic results for an unstable system. Therefore the size of a shooting segment should be small enough to get a converged solution.

To resolve the first issue, this paper uses a quadratic penalty cost function for deviation from a prescribed maneuver trajectory. Then the problem is converted into an unconstrained one, which enables us to avoid the difficulty in handling an optimal control problem with state constraints. This paper uses both linear and nonlinear helicopter motion equations, where the nonlinear one includes rotor flap and inflow dynamics. An analysis using a linear model is very effective in the software development phase because this type of analysis is much faster than that of a nonlinear model. Also the optimal solution with a linear model can be used as a good initial guess for state and costate variables, which might resolve the second issue. If we can get a large convergence radius with the above hybrid model approach, there will be a greater chance of using a high-fidelity model to identify accurate flight characteristics over a larger flight envelope. To resolve the third issue, we analyze the influence of shooting segment size on numerical convergence with a TPBVP solver. Based on this investigation, we can define the number of shooting nodes to guarantee numerical convergence. This paper is structured as follows: The next section presents the Mayer form of unconstrained nonlinear optimal control formulation. After describing the numerical procedure of MSM, the linear and nonlinear helicopter models used in the paper are summarized. Numerical analyses are carried out for bob up, turn, and slalom maneuvers. Finally, the main results of the present study are summarized in the conclusion.

\section{A Problem Statement for Optimal Control and Nec- essary Conditions}

The formulation of a general optimal control problem is covered by numerous textbooks such as Bryson and Ho, ${ }^{11)}$ Kirk, ${ }^{12)}$ and various papers. Here, we review the important results related to the present study. The optimal control problem is to find states $x^{*}(t)$, controls $u^{*}(t)$, and possibly final time $t_{\mathrm{f}}^{*}$ that minimize a cost function $J(\cdot)$. Its formulation can be written in standard Bolza form:

$$
\min J(x, u, t)=\varphi\left(x\left(t_{\mathrm{f}}\right)\right)+\int_{t_{0}}^{t_{\mathrm{f}}} f_{\mathrm{OP}}(x(t), u(t), t) d t
$$

subject to

$$
\begin{aligned}
& \dot{x}(t)=f(x(t), u(t), t) \\
& s(x(t), t) \leq 0
\end{aligned}
$$




$$
\begin{array}{ll}
x_{i}\left(t_{0}\right)=x_{i 0} & i \in I \subset\{1, \ldots, n\} \\
\psi\left(x\left(t_{\mathrm{f}}\right), t_{\mathrm{f}}\right)=0 & \psi \subseteq R^{k} \\
u(t) \in U \subseteq R^{m} & \\
t \in\left[t_{0}, t_{\mathrm{f}}\right] &
\end{array}
$$

The operating cost function $f_{\mathrm{OP}}$ usually includes the quadratic function of controls to minimize control effort. If optimal controls calculated with this kind of cost function are well within their limit, we can ignore control constraints $u(t) \in U$. One of the challenging issues in dynamic optimization is how to handle the state inequality constraint $s(x(t), t) \leq 0$. Whereas direct methods can trivially implement these constraints by using NLP, an indirect method requires complicated and tedious manipulation. When state inequality constraints are active at any time of interest, costate variables and Hamiltonian can show jumps at each touching point. Therefore, the Newton method used in MSMs, cannot be applied in a standard way. The usual remedy for this case starts by identifying the active arcs on which state constraints can be described by algebraic equality constraint equations. Then system optimality can be obtained by solving switching conditions and algebraic equations ${ }^{6)}$ together with the equations for system optimality. Consequently, the numerical procedure becomes very complex and requires prior information on the optimal trajectory, which is nearly impossible for a complex nonlinear system.

One alternative method is a penalty function based one by which the system optimality can be converted into an unconstrained optimal control problem. A logarithmic penalty function method ${ }^{13)}$ seems to be attractive because it can treat bound constraints in a more exact manner by using the barrier function method of the optimization algorithm. However, the computational speed near a constraint boundary can be prohibitively limited if we use the ODE solver with tight step size control. Also, if one of the constraints is violated during time integration, we cannot continue further analysis. There is a high probability of the occurrence of this type of situation when we use an MSM for unstable systems. This paper uses a quadratic penalty function for the trajectory deviation from a prescribed maneuver path. With the penalty cost $f_{\mathrm{SC}}$ for state constraints, optimal control formulation in the Mayer form can be rewritten as the following unconstrained one:

$$
\min J(x, u, t)=\varphi\left(x\left(t_{\mathrm{f}}\right), t_{\mathrm{f}}\right)+x_{\mathrm{CO}}\left(t_{\mathrm{f}}\right)
$$

subject to

$$
\begin{aligned}
& \dot{x}(t)=f(x(t), u(t), t) \\
& \begin{aligned}
\dot{x}_{\mathrm{CO}}(t) & =f_{\mathrm{CO}}(x(t), u(t), t) \\
& =f_{\mathrm{OP}}(x(t), u(t), t)+f_{\mathrm{SC}}(x(t), t)
\end{aligned} \\
& x_{i}\left(t_{0}\right)=x_{i 0} \\
& x_{\mathrm{CO}}\left(t_{0}\right)=0 \\
& \psi\left(x\left(t_{\mathrm{f}}\right), t_{\mathrm{f}}\right)=0
\end{aligned}
$$

The first-order necessary condition for system optimality can be derived by applying calculus of variation to the cost function. Based on the formulation in Ref. 11) and Ref. 12), optimality conditions can be expressed as the following TPBVP with state variables $y(t)=\left[x(t), x_{\mathrm{CO}}(t)\right]^{\mathrm{T}} \in R^{n+1}$, costate variables $\lambda(t) \in R^{n+1}$, Lagrange multiplier $\mu(t) \in$ $R^{k}$, and Hamiltonian $H=\lambda^{\mathrm{T}}(t) g(y(t), u(t), t)$ :

$\dot{z}(t)=\left[\begin{array}{c}\dot{y}(t) \\ \dot{\lambda}(t) \\ \dot{\mu}(t)\end{array}\right]=\left[\begin{array}{c}g(y(t), u(t), t) \\ -\left\{g_{y}(\cdot)\right\}^{\mathrm{T}} \lambda(t) \\ 0\end{array}\right]$

$r\left(z\left(t_{0}\right), z\left(t_{\mathrm{f}}\right)\right)$

$$
=\left[\begin{array}{c}
x_{i}\left(t_{0}\right)-x_{i 0} \\
x_{\mathrm{CO}}\left(t_{0}\right) \\
\psi\left(x\left(t_{\mathrm{f}}\right), t_{\mathrm{f}}\right) \\
\lambda\left(t_{\mathrm{f}}\right)-\left(\frac{\partial\left(\varphi+x_{\mathrm{CO}}\right)}{\partial y}\right)_{t=t_{\mathrm{f}}}^{\mathrm{T}}-\left(\frac{\partial \psi}{\partial y}\right)_{t=t_{\mathrm{f}}}^{\mathrm{T}} \mu
\end{array}\right]=0
$$

- Algebraic equations for optimal control:

$$
\begin{aligned}
& u^{*}(t)=\arg \min _{u} H\left(x^{*}, u, \lambda^{*}, t\right) \\
& \text { or } \frac{\partial H\left(x^{*}, u, \lambda^{*}, t\right)}{\partial u}=0
\end{aligned}
$$

\section{Numerical Methods}

A shooting algorithm ${ }^{8-10)}$ is a commonly used method of solving a TPBVP for ordinary differential equations. Since application of the single-shooting method to unstable systems like helicopters usually diverges, the following discussions focus on MSM. MSM refers to the breaking up of a trajectory into subintervals, on each of which an initial value problem is defined. The solutions are then modified through successive iterations until the boundary conditions and continuity of the state variables at the end of subintervals are satisfied. Figure 1 shows the procedures of MSM. This study follows the procedure of BNDSCO software, which has been developed by Oberle et al. ${ }^{10)}$ to solve optimal control problems with state equality and/or inequality constraints. The notation convention in Ref. 10) is used to describe the numerical procedure. First, we divide the time domain $\left[t_{0}, t_{\mathrm{f}}\right]$ into multiple subintervals to define time nodes for multiple shooting and then the following steps are applied:

(1) Define $(m+1)$ shooting nodes

$$
t_{0}:=t_{1}<t_{2}<\ldots<t_{m}:=t_{\mathrm{f}}
$$

(2) Guess initial values of $z\left(t_{j}\right)$ with

$$
Z_{j}^{(0)}, \quad j=1,2, \ldots, m
$$

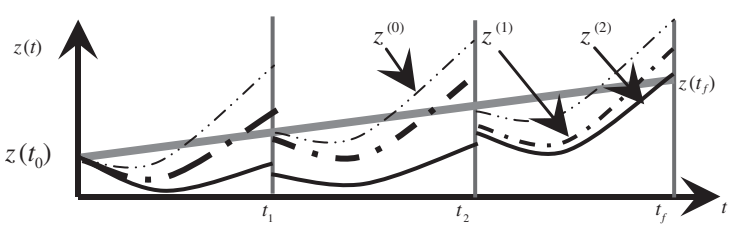

Fig. 1. Illustrative procedure of multiple-shooting method. 
(3) Iteratively solve TPBVP

(STEP 1) integrate ODE over each subinterval to find the integrated value of $z$ at time $t$ with an initial condition $z\left(t_{j}\right)=Z_{j}$ as

$$
z(t)=z\left(t ; t_{j}, Z_{j}\right) \quad t_{j} \leq t \leq t_{j+1}
$$

(STEP 2) Compute jumps $F_{j}$ at each shooting node $(1 \leq j \leq m-2)$

$F_{j}\left(Z_{1}, Z_{2}, \ldots, Z_{m-1}\right):=z\left(t_{j+1} ; t_{j}, Z_{j}\right)-Z_{j+1}$

and evaluate the residual in boundary conditions

$$
F_{m-1}\left(Z_{1}, Z_{2}, \ldots, Z_{m-1}\right):=r\left(Z_{1}, Z_{m-1}\right)
$$

(STEP 3) Define an approximate Jacobean matrix

$$
M \text { of } F\left(Z_{1}, Z_{2}, \ldots, Z_{m-1}\right)
$$

(STEP 4) Solve the matrix equation to find the corrections $\Delta Z$ for states and costates

$$
M \Delta Z=-F\left(Z_{1}, Z_{2}, \ldots, Z_{m-1}\right)
$$

(STEP 5) Update the state and costate variables

$$
Z^{\text {new }}=Z+\alpha \Delta Z \quad \alpha \in[0,1]
$$

The time integration routine in (STEP 1) and (STEP 4) is the innermost part of the iterative MSM and the computation time of this routine determines the overall efficiency of numerical analysis. For time integration, first, we should solve Eq. (7) to get optimal controls which requires approximation of the Hessian matrix $H_{\mathrm{uu}}$ and gradient $H_{\mathrm{u}}$. If system equations are affine in controls and a quadratic cost function is used for control with positive definite weighting matrix $R$,

$$
\begin{aligned}
& \text { affine system: } \dot{x}(t)=f_{1}(x(t), t) u(t)+f_{2}(x(t), t) \\
& \text { cost for control: } \int_{t_{0}}^{t_{1}} 0.5\left(u-u_{\text {trim }}\right)^{\mathrm{T}} R\left(u-u_{\text {trim }}\right) d t
\end{aligned}
$$

then we can approximate the Hessian $H_{\mathrm{uu}}$ with $\lambda_{\mathrm{CO}} R$ to reduce computation time. It can also be a good approximation for the system nearly affine in the control, which is the usual case in helicopter dynamic equations. The state gradient $H_{\mathrm{x}}$ and the control gradient $H_{\mathrm{u}}$ are numerically approximated by a central differencing method, which gives better numerical accuracy and stability than one-sided differencing.

\section{Mathematical Model}

\subsection{Helicopter flight dynamic equations}

The helicopter flight dynamic models in this paper are based on previous research results of Ref. 14), where rotor dynamic equations for flap, lead-lag, and RPM dynamics have been formulated in fully implicit form. A rotor was modeled with rigid blades with spring and damper. Nonlinear quasi-steady aerodynamic theory has been applied through table look-up. Trim calculations for the Bo105 helicopter have been carried out using a partial periodic trimming algorithm in which a DAE solver was used for time integration. The linear model used in this paper is derived by linearizing helicopter motion equations at a calculated trim condition. A state equation is approximated in time invariant form by averaging both state and control derivative matrices over one rotor revolution. The nonlinear model has been derived by the using linear aerodynamic theory rather than the nonlinear one in Ref. 14). The helicopter models, used in this study, can be summarized as:

(1) 6-DOF linear model (only rigid body DOF)

(2) 12-DOF linear model

(3) 12-DOF nonlinear model

The states and controls for the 12-DOF model can be defined with the states for rigid body, dynamic inflow, main rotor flap, and controls as

$$
\begin{aligned}
x & =\left[x_{\mathrm{R}}, x_{\mathrm{I}}, x_{\mathrm{F}}\right]^{\mathrm{T}} \\
x_{\mathrm{R}} & =\left[u, v, w, p, q, r, \phi, \theta, \psi, x_{\mathrm{E}}, y_{\mathrm{N}}, h\right]^{\mathrm{T}} \\
x_{\mathrm{I}} & =\left[\lambda_{0}, \lambda_{1 \mathrm{C}}, \lambda_{1 \mathrm{~S}}\right]^{\mathrm{T}} \\
x_{\mathrm{F}} & =\left[\beta_{0}, \beta_{1 \mathrm{C}}, \beta_{1 \mathrm{~S}}\right]^{\mathrm{T}} \\
u & =\left[\delta_{0}, \delta_{1 \mathrm{C}}, \delta_{1 \mathrm{~S}}, \delta_{\mathrm{TR}}\right]^{\mathrm{T}}
\end{aligned}
$$

\subsection{Description of maneuver trajectory}

Generation of the trajectory is one of the most important topics in recent research. The main reason is its use in vehicle motion planners for guidance and control of autonomous air vehicles. High-level flight functions, such as obstacle avoidance and terrain following, etc., can be implemented with a trajectory generated in optimal ways. The trajectory description with polynomials and trigonometric functions is widely used in studies on inverse simulation of the helicopter because of its analytical simplicity and smoothness. ${ }^{4,5,15)}$ The feasibility of a generated trajectory can be enhanced by correlating it with the results of flight tests. In research in this area, the piecewise continuous polynomial approach of Thompson et al. ${ }^{15)}$ is very attractive because their method provides a simple yet effective way of modeling the maneuver aggressiveness as required. The generated trajectory showed good agreement with flight test data and has been used to predict a handling quality requirement for roll-quickness parameters. ${ }^{15)}$ This paper uses similar analytical descriptions to those for inverse simulation.

The trajectory can be expressed as the sum of states at maneuver entry and its variation during maneuver.

$$
\begin{aligned}
& x(t)=x\left(t_{\text {entry }}\right)+\Delta x(t) \\
& \text { or } x(t)=x\left(t_{\text {entry }}\right)+\int_{t_{\text {entry }}}^{t} \Delta \dot{x}(\tau) d \tau
\end{aligned}
$$

Trigonometric functions can be good basis functions for trajectory generation ${ }^{1)}$ because of their smoothness. In this paper, the trajectories of bob up and slalom maneuvers are generated with Eq. (14) and Eq. (15), respectively.

$$
\begin{aligned}
& \Delta x(\bar{t})=\frac{(\Delta x)_{\max }}{16.0}[8+\cos (3 \pi \bar{t})-9 \cos (\pi \bar{t})] \\
& \Delta x(\bar{t})=\frac{(\Delta x)_{\max }}{46.8}\left[\begin{array}{l}
32+\sin (2 \pi \bar{t})-20 \sin (4 \pi \bar{t}) \\
+2 \sin (8 \pi \bar{t})
\end{array}\right]
\end{aligned}
$$




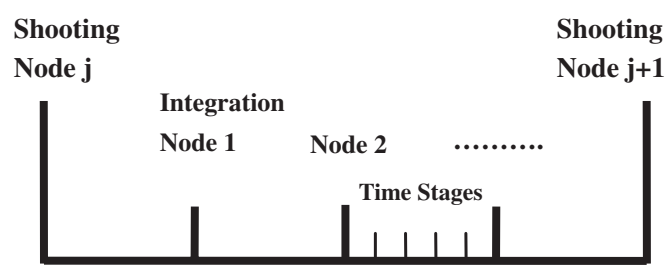

Fig. 2. Distribution of computational nodes.

where

$$
\bar{t}=\left(t-t_{\text {entry }}\right) /\left(t_{\text {finish }}-t_{\text {entry }}\right) \quad 0 \leq \bar{t} \leq 1
$$

The piecewise polynomial method proposed by Thompson et al. ${ }^{15)}$ can generate a trajectory as shown in Fig. 2. A typical maneuver can be described by its different maneuver phases such as entry stage, steady maneuver phase, and exit phase. By changing the time required for the entry phase and the exit phase, we can control the level of maneuver aggressiveness. A turning flight with a constant turn rate is an example of the steady maneuver phase. Here we apply this method to trajectory generation for a turn maneuver with following mathematical description:

$$
\begin{aligned}
& \Delta \dot{x}(t)=\left\{\begin{array}{cc}
(\Delta \dot{x})_{\max }\left(-2 \bar{t}^{3}+3 \bar{t}^{2}\right), & t_{\text {entry }} \leq t \leq t_{\text {steady }} \\
(\Delta \dot{x})_{\max }, & t_{\text {steady }} \leq t \leq t_{\text {exit }} \\
(\Delta \dot{x})_{\max }\left(-2 \tilde{t}^{3}+3 \tilde{t}^{2}\right), & t_{\text {exit }} \leq t \leq t_{\text {finish }}
\end{array}\right. \\
& (\Delta \dot{x})_{\max }=(\Delta x)_{\max } /\left(t_{\text {finish }}+t_{\text {exit }}-t_{\text {steady }}-t_{\text {entry }}\right) \\
& \bar{t}=\left(t-t_{\text {entry }}\right) /\left(t_{\text {steady }}-t_{\text {entry }}\right) \\
& \tilde{t}=1-\left(t-t_{\text {exit }}\right) /\left(t_{\text {finish }}-t_{\text {exit }}\right)
\end{aligned}
$$

Because a maneuver is always carried out in $3 \mathrm{D}$ space, it needs a description in all axes. Most maneuvers, defined in modern handling quality requirements, start the maneuver from a stabilized trim condition and end it with the other steady flight condition. The requirements for maneuver accuracy are commonly specified with the boundary deviation from a constant reference value in flight speed, altitude, sideslip, heading, and positions, etc. In the same way, this paper uses these quantities to define the reference trajectory for other axes.

\subsection{Cost function and boundary conditions}

The quadratic cost function for control is commonly used in dynamic optimization, and the system limits are usually described by state constraints, control constraints, and with any suitable form of performance limitation. As previously mentioned, the numerical implementation of a state constraint is very difficult with the indirect method. In this paper, state constraints are considered with a quadratic penalty function as the following:

$$
\begin{aligned}
f_{0}\left(\bar{x}_{\mathrm{R}}(t), u(t), t\right)= & 0.5\left(\bar{x}_{\mathrm{R}}-\bar{x}_{\text {target }}\right)^{\mathrm{T}} Q\left(\bar{x}_{\mathrm{R}}-\bar{x}_{\text {target }}\right) \\
& +0.5\left(u-u_{\text {trim }}\right)^{\mathrm{T}} R\left(u-u_{\text {trim }}\right)
\end{aligned}
$$

where,

$$
\begin{aligned}
& \bar{x}_{\mathrm{R}}: \text { reduced rigid body states } \\
& \bar{x}_{\text {target }}: \text { target states } \\
& \bar{x}_{\mathrm{R}}(t)=\left[u, v, w, p, q, \dot{\psi}, \phi, \theta, \psi, x_{\mathrm{E}}, y_{\mathrm{N}}, h\right]^{\mathrm{T}} \\
& R=\operatorname{diag}\left(r_{\delta_{0}}, r_{\delta_{1 \mathrm{C}}}, r_{\delta_{1 \mathrm{~S}}}, r_{\delta_{\mathrm{TR}}}\right)
\end{aligned}
$$

Table 1. Diagonal components of state weighting matrix.

\begin{tabular}{cccccc}
\hline$q_{u}$ & $q_{v}$ & $q_{w}$ & $q_{p}$ & $q_{q}$ & $q_{\dot{\psi}}$ \\
200 & 7.5 & 4.5 & 7.5 & 15 & 200 \\
\hline$q_{\phi}$ & $q_{\theta}$ & $q_{\psi}$ & $q_{x \mathrm{E}}$ & $q_{y \mathrm{~N}}$ & $q_{h}$ \\
100 & 500 & 35 & 0 & 100 & 30 \\
\hline \multicolumn{5}{c}{ Note: The components are the same for all MTEs except } \\
& 1. $q_{q}=40$ for side step \\
2. & $q_{\dot{\psi}}=400$ and $q_{\psi}=350$ for turn \\
3. & $q_{y \mathrm{~N}}=0$ for turn
\end{tabular}

$Q=\operatorname{diag}\left(q_{u}, q_{v}, q_{w}, q_{p}, q_{q}, q_{\dot{\psi}}, q_{\phi}, q_{\theta}, q_{\psi}, q_{x \mathrm{E}}, q_{y \mathrm{~N}}, q_{h}\right)$

The target states $\bar{x}_{\text {target }}(t)$ are set to be trim states $\left(x_{\mathrm{R}}\right)_{\mathrm{TRIM}}$ except those which need the description of their time variation for a specific maneuver. The same control weight matrix $R$ is used throughout this paper with its diagonal components as:

$$
r_{\delta_{0}}=3600, \quad r_{\delta_{1 \mathrm{C}}}=900, \quad r_{\delta_{1 \mathrm{~S}}}=900, \quad r_{\delta_{\mathrm{TR}}}=600
$$

The positive semi-definite weight matrix $Q$ has its components are listed in Table 1. The components show small variation in their value, depending on the required tracking accuracy for a specific maneuver.

The initial conditions for state variables can be specified with the results of trim analysis because maneuvers in this study are started from a steady trim condition and their terminal conditions can be defined with target states at the end of a maneuver.

$$
\begin{aligned}
x_{i}\left(t_{0}\right) & =x_{i, \text { trim }}, \quad i=1, \ldots, n \\
\bar{x}\left(t_{\mathrm{f}}\right) & =\bar{x}_{\text {target }}\left(t_{\mathrm{f}}\right)
\end{aligned}
$$

Since the penalized cost function already considers the same conditions, we can use the implementation of terminal constraints both for the exact matching of our trajectory with a target trajectory at the final time and for providing the improved convergence of MSM. The terminal cost function can be set to zero if time optimal problems are excluded.

\section{Applications}

We can apply the present approaches to representative helicopter maneuvers such as bob up, turn, and slalom, and the Bo-105 helicopter configuration has been used as a mathematical model. The bob up and side step enter into the maneuver from hover and the turn maneuver from steady level flight at a forward speed of 60 knots. Table 2 shows the main parameters for each maneuver calculated in this study. Time integration is carried out using the 4-stage RungeKutta method with fixed time-step size and time integration nodes between two adjacent shooting nodes distributed as shown in Fig. 2. The number of time integration nodes can be selected to control the time step size or to improve time integration accuracy. If not specifically mentioned, we use an impulsive start method where state and costate variables over all shooting nodes are initialized with the same values. In this study, the trim values are used to initialize state variables, and costate variables are simply set to zero except the one corresponding to cost equation with 1.0. 
Table 2. Parameters for maneuver description.

\begin{tabular}{cccc}
\hline Maneuvers & $\begin{array}{c}(\Delta x)_{\max } \text { in m, } \\
\text { deg, deg } / \mathrm{sec}\end{array}$ & $t_{\text {entry }}(\mathrm{sec})$ & $t_{\text {exit }}(\mathrm{sec})$ \\
\hline Bop up & $(\Delta h)_{\max }=15$ & 2.0 & 8.0 \\
Turn $^{(1)}$ & $(\Delta \psi)_{\max }=90$ & 2.0 & 12.0 \\
Slalom & $(\Delta y)_{\max }=10$ & 1.0 & 9.0 \\
\hline
\end{tabular}

Note (1): $t_{\text {steady }}=5.0 \mathrm{sec}, t_{\text {exit }}=9.0 \mathrm{sec}$ for turn.

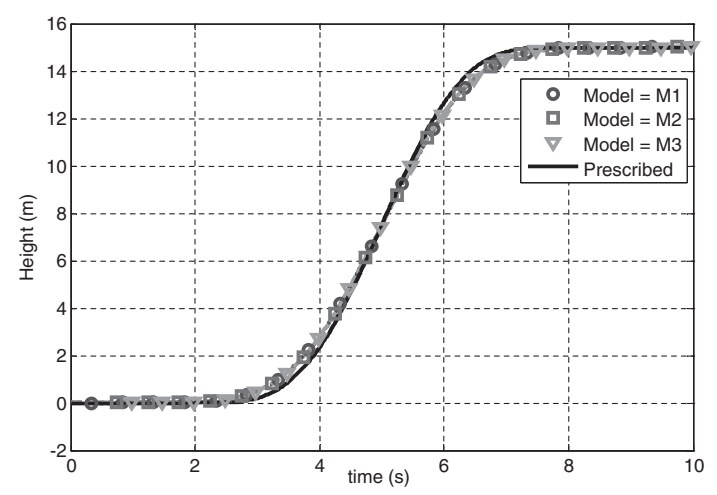

Fig. 3. Height variation during bob up maneuver with different helicopter models.

For simplicity, the analysis conditions are defined with the notation of Mx-NSy-NIz, where Mx, NSy, and NIz are used to differentiate the mathematical model used, the number of shooting nodes, and the number of integration nodes, respectively. The three different models used in this study are classified as:

$$
\begin{aligned}
& \text { M1 }=1: \text { Linear 6-DOF model }(x=1) \\
& \text { M2 }=2: \text { Linear 12-DOF model }(x=2) \\
& \text { M3 }=3: \text { Nonlinear 12-DOF model }(x=3)
\end{aligned}
$$

For example, the notation M3-NS3000-NI6 defines an analysis condition when we use a nonlinear twelve degree of freedom model with 3000 multiple shooting nodes and six time integration nodes.

Figures 3, 4, and 5 show the comparisons between the prescribed trajectories and the present results with variations of the helicopter mathematical model. The different models calculate nearly the same trajectory, and the calculated trajectories match well with the prescribed paths except the heading rate for a turn maneuver. Simultaneous application of both heading angle and heading rate for the turn trajectory is arguable but this kind of approach generally improves numerical stability. Figure 6 presents the convergence history when MSM is implemented to the bop up maneuver with a varying number of shooting nodes at the M1-I6 analysis condition. The convergence speed is reduced remarkably by a small number of shooting nodes, and the analysis failed to converge with less than 40 shooting nodes. The same tendency can be found in Fig. 7 with the M2-NI6 analysis condition. The inclusion of flap and inflow dynamics adversely affects convergence. Figure 8 shows the convergence history at the M1-NS50 analysis condition with a varying number

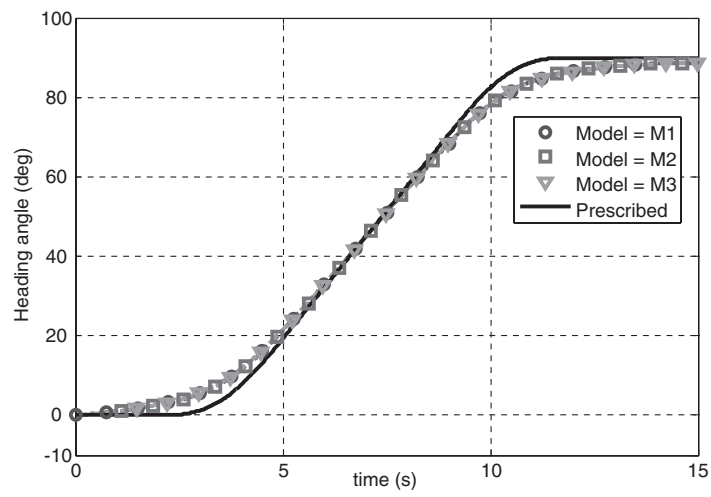

(a) Heading angle

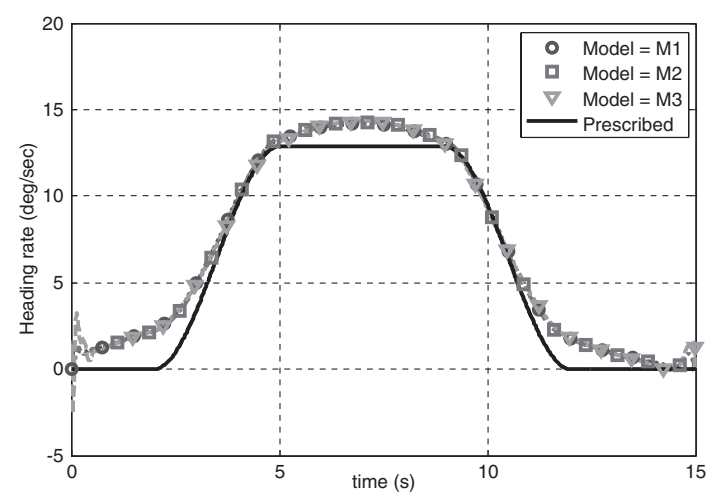

(b) Heading angular rate

Fig. 4. Heading angle and heading angular rate change at turn maneuver with different helicopter models.

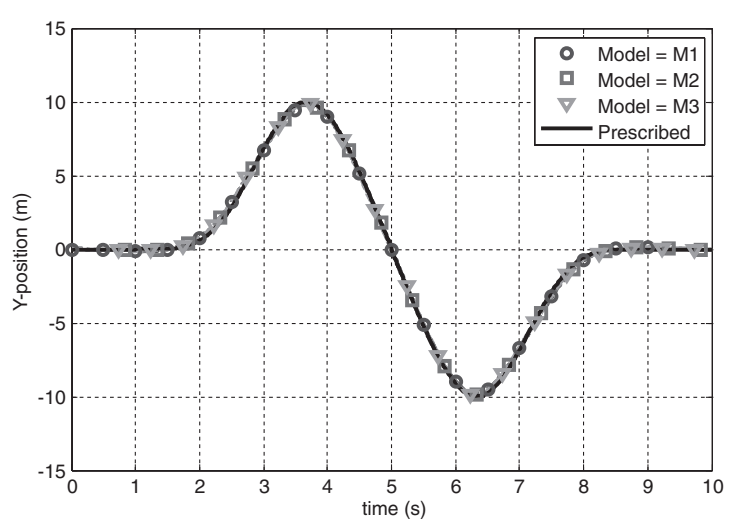

Fig. 5. Y-position change during slalom maneuver with different helicopter models.

of integration nodes, showing the dependency of numerical convergence on time integration error. Since the total number of time integration stages is proportional to the product of the number of shooting nodes and the number of integration nodes, we need a trade-off between these two parameters to get both good convergence and numerical efficiency. Figure 9 differentiates the convergence histories with different methods of initializing state and costate variables at multiple shooting nodes. Generally, the convergence speed of MSM is very slow at early iterative stages due to poor initial guesses. Once the calculated trajectory approaches the converged optimal one, the absolute error tolerance starts to de- 


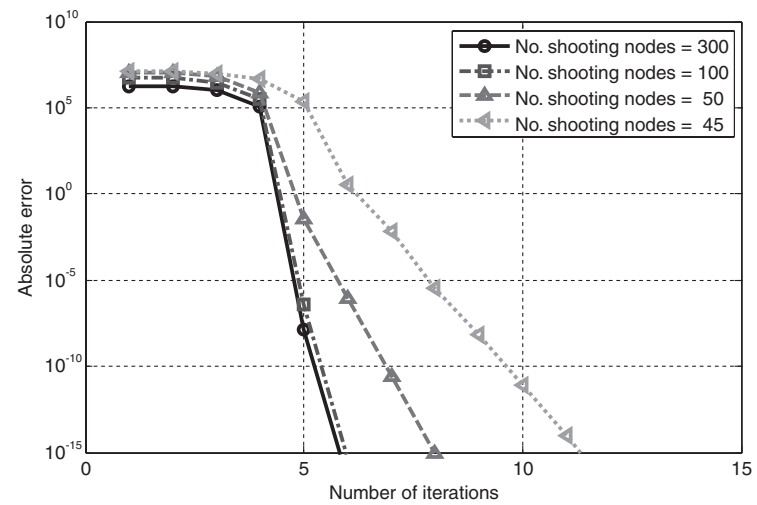

Fig. 6. MSM convergence history for bob up maneuver. (Effect of number of shooting nodes: M1-NI6.)

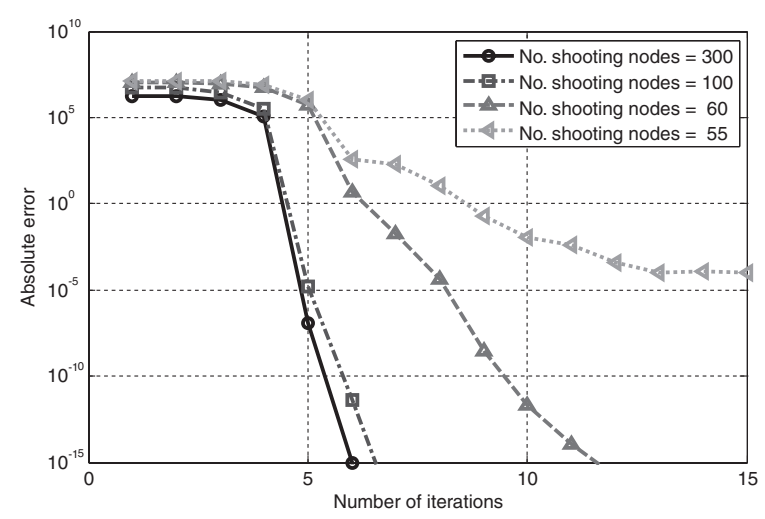

Fig. 7. MSM Convergence history for bob up maneuver. (Effect of number of shooting nodes: M2-NI6.)

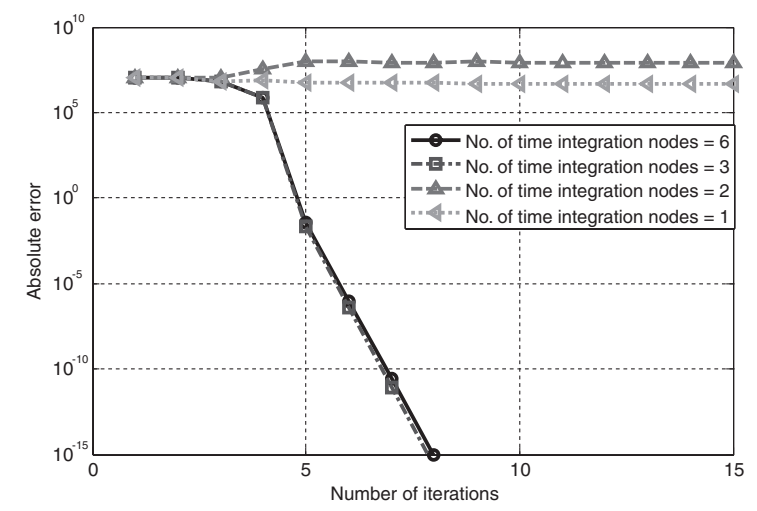

Fig. 8. MSM Convergence history for bob up maneuver. (Effect of number of time integration nodes: M1-NS50.)

crease rapidly. By using optimal calculations with the M1 model as initial guesses of state and costate variables for the same analysis with the M2 model, we get accelerated convergence. Also this approach is very effective in guaranteeing the numerical convergence of the applications with nonlinear model. Although an impulsive start with the M3 model fails to converge, we can get a converged solution by using the optimal calculations with the M1 or M2 model as initial guesses for the M3 model.

Figure 10 presents calculated costate variables for turn

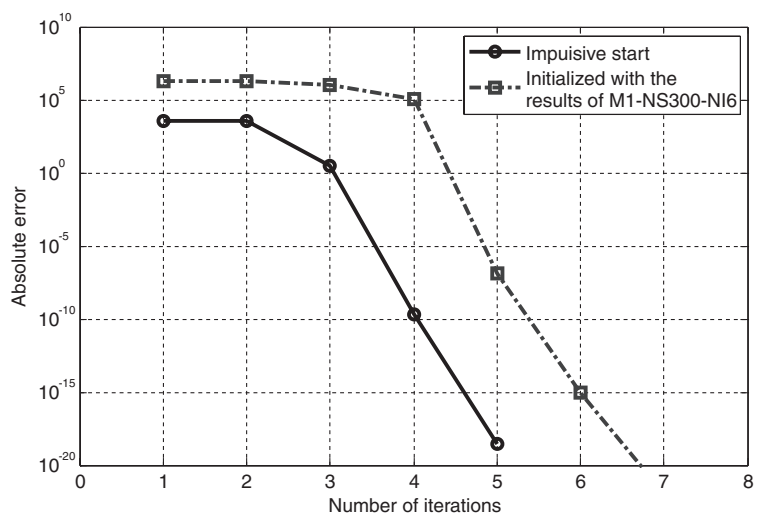

Fig. 9. MSM convergence history for bob up maneuver. (Effect of initialization for M2-NS300-NI6.)

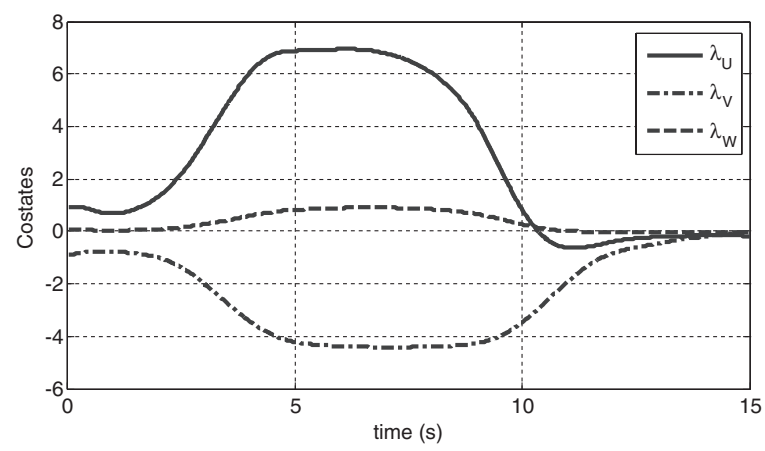

(a) 6-DOF linear model

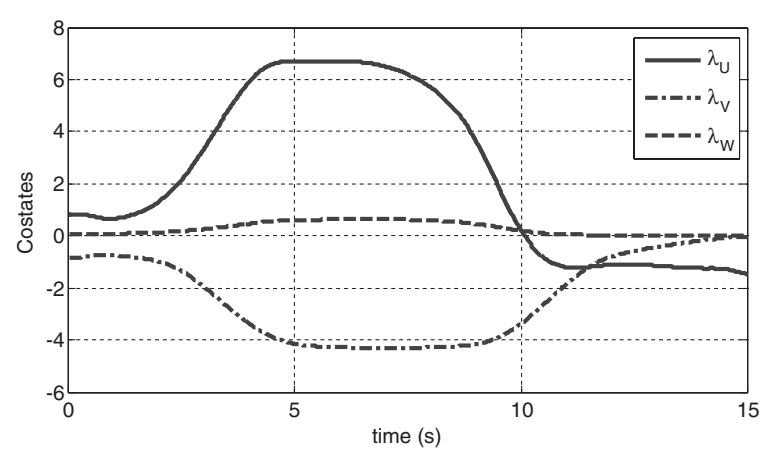

(b) 12-DOF linear model

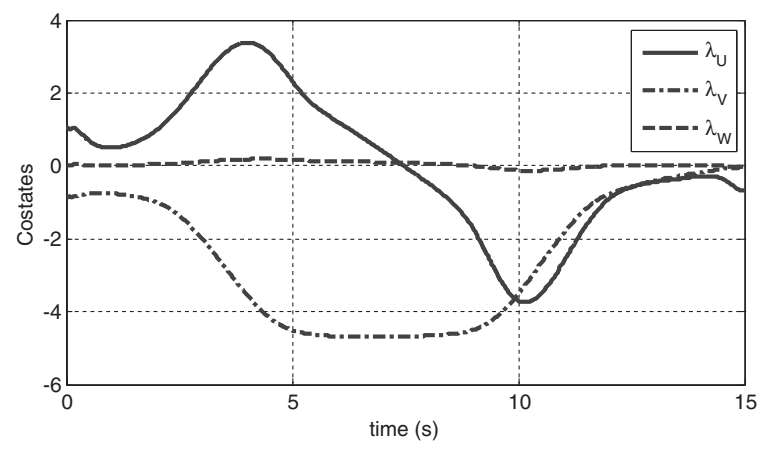

(c) 12-DOF nonlinear model

Fig. 10. Calculated costate variables for turn maneuver with different models.

maneuvers with different models. The calculated costate variable for longitudinal velocity with a nonlinear model differs noticeably from those with two linear models. The 

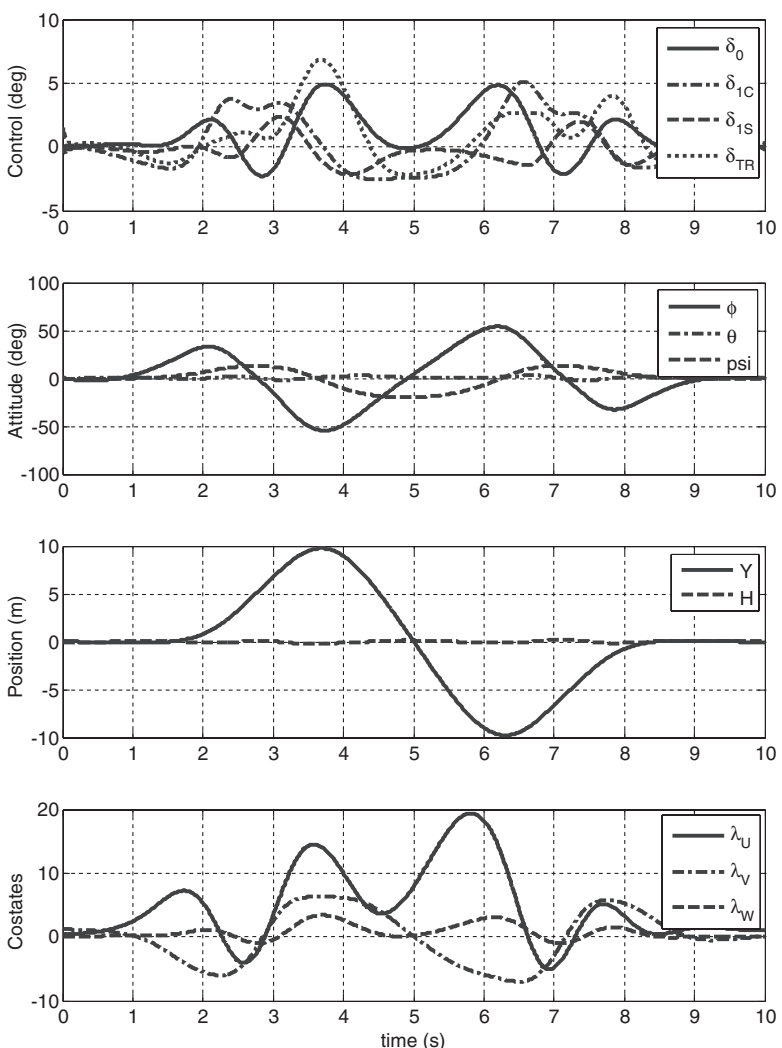

Fig. 11. Optimal trajectory for slalom maneuver with 6-DOF linear model (M1).

other variables show similar results. In this case, initialization only with state variables calculated by the M1 or M2 model is sufficient to get the desired convergence in the analysis with the M3 model.

The next analysis is for the slalom maneuver, which is a complex multi-axial maneuver. Figures 11, 12, and 13 show the numerical results for analysis conditions M1-NS1500NI6, M2-NS1500-NI6, and M3-NS1500-NI6, respectively. Present analysis with the M3 model uses optimal state variable, calculated with the M1 model, to initialize the state variables at shooting nodes and applies the impulsive start method for costate variables. The converged solutions are obtainable when more than 1500 shooting nodes is used. Otherwise, the most solution fails to converge. The state variables show good correspondence but some costate variables, calculated with the M3 model, present a noticeable difference from the results calculated with the M1 or M2 model, which might validate the present choice of the initialization method for the M3 model.

\section{Conclusions}

This paper has studied implementation issues of the indirect method to helicopter inverse simulation, which includes numerical difficulties in handling active state constraints, the problem of small convergence radii due to poor initial guesses, and issues related to its application to unstable systems.

Application to helicopter maneuver analyses show the
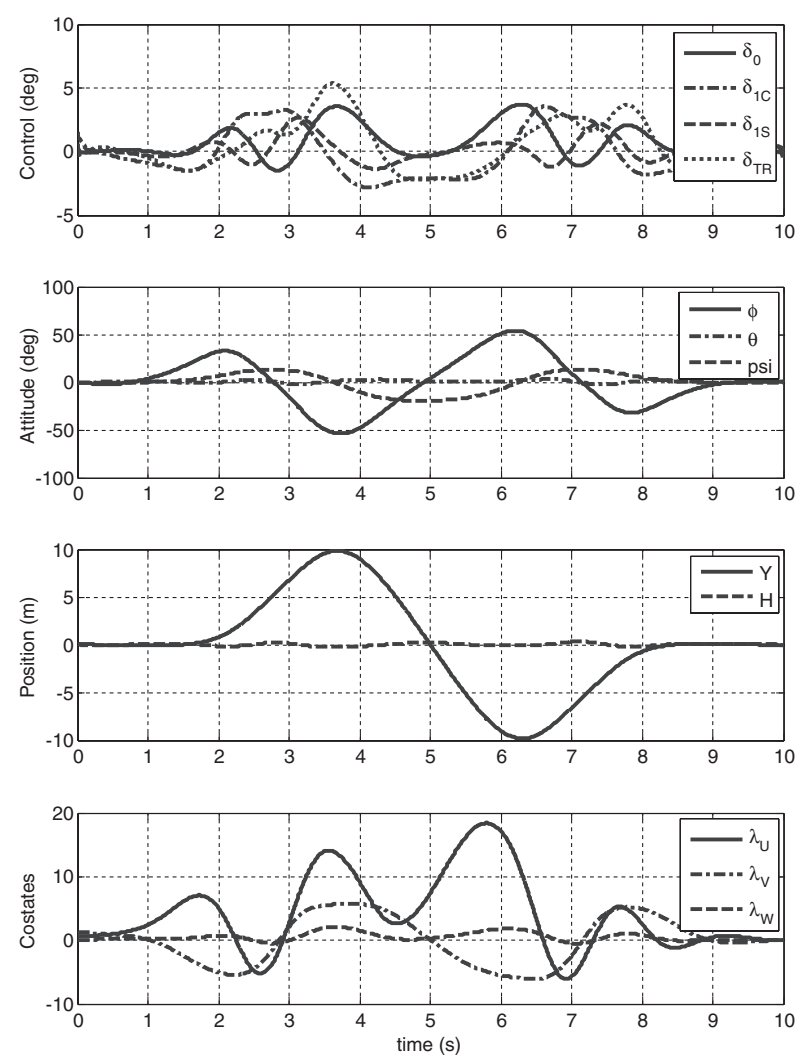

Fig. 12. Optimal trajectory for slalom maneuver with 12-DOF linear model (M2).
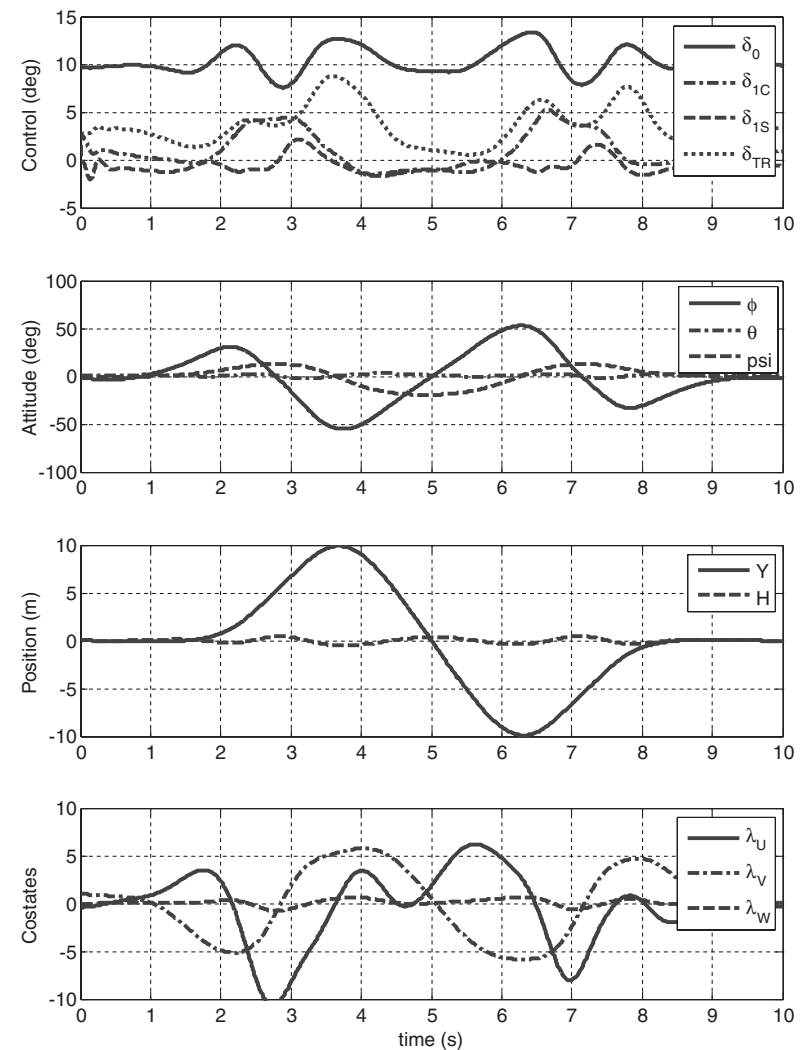

Fig. 13. Optimal trajectory for slalom maneuver with 12-DOF nonlinear model (M3). 
predicted optimal trajectory tracked the prescribed one well, which proves the validity of the penalty function approach. Also, if optimal solutions with the linear model are used to initialize state and costate variables for the analysis with the nonlinear model, the analysis with nonlinear model offers the increased convergence radius and fast convergence speed. Consequently, the present hybrid model approach provides good initial guesses for optimal control solutions with high-fidelity helicopter model. Finally, the indirect method could be applicable to optimal control problems for unstable helicopter systems by selecting more than the minimum required number of shooting nodes.

Although the numerical approach has been applied to maneuver analysis of helicopters, possible applications are not limited to this specific topic. The main results can provide a valuable motivation for applying the indirect method to the numerical analysis of various nonlinear helicopter flight mechanics problems.

\section{Acknowledgments}

This work was supported by the faculty research fund of Konkuk University in 2006.

\section{References}

1) Hess, R. A. and Gao, C.: A Generalized Algorithm for Inverse Simulation Applied to Helicopter Maneuvering Flight, J. Am. Helicopter Soc., 16, October (1993), pp. 3-14.
2) Avanzini, G. and de Matteis, G.: Two-Timescale Inverse Simulation of a Helicopter Model, J. Guid. Control Dynam., 24 (2001), pp. 330-339.

3) Celi, R.: Optimization-Based Inverse Simulation of a Helicopter Slalom Maneuver, J. Guid. Control Dynam., 23 (2000), pp. 289-297.

4) Rutherford, S. and Thomson, D. G.: Improved Methodology for Inverse Simulation, Aeronaut. J., 100 (1996), pp. 79-86.

5) Bradley, R. and Thomson, D. G.: The Development of Inverse Simulation for Quantitative Assessment of Helicopter Handling Qualities, Aeronaut. J., 101 (1997), pp. 287-294.

6) Cervantes, L. and Biegler, L. T.: Optimization Strategies for Dynamic Systems, Encyclopedia of Optimization, 5 (2001), pp. 400-413.

7) Bottasso, C. L., Croce, A., Leonello, D. and Riviello, L.: Rotorcraft Trajectory Optimization with Realizability Considerations, J. Aerospace Eng., 18, 3 (2005), pp. 146-155.

8) Sim, Y. C., Leng, S. B. and Subramaniam, V.: A Combined Genetic Algorithms-Shooting Method Approach to Solving Optimal Control Problems, Int. J. Syst. Sci., 31, 1 (2000), pp. 83-89.

9) Fraser-Andrews, G.: A Multiple-Shooting Technique for Optimal Control, J. Optim. Theory Appl., 102, 2 (1999), pp. 299-313.

10) Oberle, H. J. and Grimm, W.: BNDSCO; A Program for the Numerical Solution of Optimal Control Problems, DFVLR Report No. 515, 1989.

11) Bryson, A. E. Jr. and Ho, Y. C.: Applied Optimal Control, Hemisphere Publishing Corporation, Washington, D.C., 1975.

12) Kirk, D. E.: Optimal Control Theory; An Introduction, Dover Publications, Inc., New York, 1970.

13) Bonnans, J. F. and Guilbaud, Th.: Using Logarithmic Penalties in the Shooting Algorithm for Optimal Control Problems, Optimal Control Applications and Methods, 24 (2003), pp. 257-278.

14) Kim, C.-J., Yun, C. Y. and Choi, S.: Fully Implicit Formulation and Its Solution for Rotor Dynamics by Using Differential Algebraic Equation (DAE) Solver and Partial Periodic Trimming Algorithm (PPTA), 31st European Rotorcraft Forum, Italy, 2005.

15) Thompson, D. G. and Bradley, R.: Mathematical Definition of Helicopter Maneuvers, J. Am. Helicopter Soc., 42 (1997), pp. 307-309. 